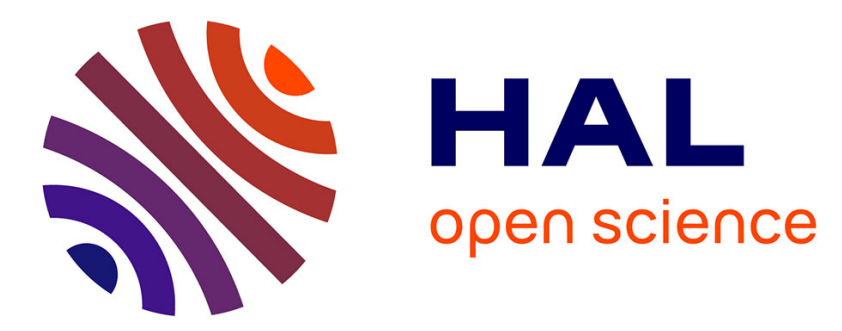

\title{
Contributions sur la transformation numérique (Editorial)
}

François de Corbière, Cécile Godé, Jessie Pallud

\section{To cite this version:}

François de Corbière, Cécile Godé, Jessie Pallud. Contributions sur la transformation numérique (Editorial). Systèmes d'Information et Management, 2019, 24 (2), pp.3-5. 10.3917/sim.192.0003 . hal-02317391

\section{HAL Id: hal-02317391 \\ https://hal.science/hal-02317391}

Submitted on 16 Oct 2019

HAL is a multi-disciplinary open access archive for the deposit and dissemination of scientific research documents, whether they are published or not. The documents may come from teaching and research institutions in France or abroad, or from public or private research centers.
L'archive ouverte pluridisciplinaire HAL, est destinée au dépôt et à la diffusion de documents scientifiques de niveau recherche, publiés ou non, émanant des établissements d'enseignement et de recherche français ou étrangers, des laboratoires publics ou privés. 


\section{Éditorial}

\section{CONTRIBUTIONS SUR LA TRANSFORMATION NUMÉRIQUE}

François de Corbière, Cécile Godé et Jessie Pallud

ESKA | «Systèmes d'information \& management »

2019/2 Volume 24 | pages 3 à 5

ISSN $1260-4984$

ISBN 9782747229203

Article disponible en ligne à l'adresse :

https://www.cairn.inforevue-systemes-d-information-etmanagement-2019-2-page-3.htm

Distribution électronique Cairn.info pour ESKA.

(C) ESKA. Tous droits réservés pour tous pays.

La reproduction ou représentation de cet article, notamment par photocopie, n'est autorisée que dans les limites des conditions générales d'utilisation du site ou, le cas échéant, des conditions générales de la licence souscrite par votre établissement. Toute autre reproduction ou représentation, en tout ou partie, sous quelque forme et de quelque manière que ce soit, est interdite sauf accord préalable et écrit de l'éditeur, en dehors des cas prévus par la législation en vigueur en France. Il est précisé que son stockage dans une base de données est également interdit. 


\section{Éditorial}

\section{Contributions sur la transformation numérique}

\section{François DE CORBIÈRE, Cécile GODÉ E Jessie PALLUD}

Dans cet éditorial, l'équipe des rédacteurs a choisi de faire un retour sur la conférence de l'AIM 2019 et de mettre en lumière sa résonnance avec la politique éditoriale de SIM. Le thème de la conférence sur « La transformation numérique des organisations » a permis à notre communauté de présenter des communications et d'entamer des discussions scientifiques sur différentes facettes de la transformation numérique. Nous retenons plus particulièrement nos keynote speakers, Yves Pigneur et Lynne Markus.

Sur la thématique «Innover et repenser son modèle d'affaires », Pigneur (2019) souligne l'importance pour les entreprises d'opérer une transformation numérique de leurs activités, et notamment de leurs modèles d'affaires, pour générer de la valeur et rester innovantes. Il nous a notamment présenté des exemples de réussite et d'échec de modèles d'affaires d'entreprises en transformation numérique. Un constat fort intéressant est que les entreprises qui réussissent le mieux de nos jours sont celles qui gèrent non pas un modèle d'affaires unique, mais plutôt des portefeuilles de modèles d'affaires qui incluent des activités à rendement plus ou moins faible et représentant un risque plus ou moins élevé. La clé du succès réside alors dans cette capacité à pouvoir faire évoluer les différents modèles d'affaires d'un même portefeuille selon ces deux axes. Acquérir, améliorer un modèle d'affaires ou au contraire désinvestir dans les modèles d'affaires qui n'ont pas d'avenir, un art que bien des entreprises ont encore du mal à maîtriser !

Dans une perspective plus sociétale, Markus (2019) nous a incité à développer des programmes de recherche sur les conditions d'émergence de la transformation numérique. Lynne Markus explique que chaque transformation est unique et complexe car de nombreux éléments sont à l'œuvre (sociaux, économiques, technologiques, réglementaires...) et la chaîne de causes à effet peut être aussi très longue, limitant ainsi les conditions de généralisation des résultats. Ainsi, les chercheurs devraient s'appuyer sur plusieurs cas afin de comparer les contextes et de développer une compréhension plus approfondie du rôle des technologies dans cette transformation. Elle encourage également les chercheurs à étudier des innovations digitales qui soient liées à des transformations sociétales, telles que l'introduction de la monnaie mobile en Afrique Subsaharienne dont l'impact dépasse la seule frontière technologique.

La transformation numérique n'est pas un sujet nouveau pour les chercheurs en management des systèmes d'information 
puisque, déjà en 2002, Robert Reix ${ }^{1}$ affirmait que "les technologies de l'information (T.I.) sont à la mode, et cette mode persiste! Persiste aussi le discours dominant sur leur capacité à transformer les organisations". Néanmoins, les publications académiques sur ce sujet sont en forte croissance. Le fort potentiel de transformation et de reconfiguration (Orlikowski et Scott, 2016 ; Faraj et al., 2018) des technologies numériques sur les organisations incite donc les chercheurs à développer des travaux scientifiques dans ce champ. Ainsi, dans les grandes revues de notre discipline, la transformation numérique s'ancre avec des articles ou des numéros spéciaux autour de différentes thématiques : la transformation du travail (Eden et al., 2019), l'entrepreneuriat (Li et al., 2018), les problématiques des DSI (Kappelman et al., 2019), le management de l'innovation (Nambisan et al., 2017), les modèles d'affaires basés sur les plateformes (Constantinides et al., 2018), la chaîne de valeur (Kauffman et Weber, 2018), l'alignement stratégique (Denner $e t$ al., 2018)... Ce sujet n'est pas en reste dans la sphère organisationnelle et managériale. En effet, la transformation digitale est une préoccupation majeure des entreprises. Selon une étude de Haussman Executive Search $^{2}$, 90\% des entreprises du CAC40 ont d'ailleurs nommés un Chief Digital Officer (CDO) au sein de leurs comités exécutifs afin de les accompagner dans leur transformation.

L'analyse des processus de transformation du travail et des compétences, des structures organisationnelles, de la gouvernance, du management ou des modèles d'affaires associés au numérique est au coeur de notre discipline, et nombreuses sont les recherches publiées dans SIM qui ont contribué au développement de connaissances scientifiques sur le sujet. SIM se présente ainsi comme un forum de discussion autour de la transformation numérique et de ses implications humaines, managériales, organisationnelles, voire sociétales comme nous le démontrait Markus en juin dernier. Cette question des processus transformationnels est transversale, transcendant les frontières disciplinaires. Notre communauté est à la pointe des recherches dans le domaine, mais d'autres disciplines, tels que le marketing, la logistique et Supply Chain, la stratégie et l'innovation, les ressources humaines ou encore le management de la santé contribuent à améliorer nos connaissances en développant des travaux d'intérêt sur la question. Chères lectrices et chers lecteurs, nous attendons avec impatience vos propositions de contributions sur la transformation numérique, ses processus et ses effets performatifs et transformatifs dans les organisations!

Dans ce numéro, nous retrouvons trois contributions sur la transformation numérique.

Muriel Mignerat, Laurent Mirabeau et Karine Proulx s'intéressent à la transformation numérique des pratiques individuelles via l'usage des technologies de l'information dans une université canadienne. Par la mobilisation de la théorie institutionnelle et l'analyse des comportements stratégiques autonomes mis en ouvre par les acteurs, les auteurs mettent en exergue les facteurs et mécanismes favorisant l'émergence du BYOD (Bring Your Own Device).

Nabila Boukef et Mohamed-Hédi Charki apportent une contribution sur la transformation numérique à l'heure où les Réseaux Sociaux d'Entreprises (RSE) sont de plus en plus présentés comme une alternative à l'usage de l'email. Par une approche enracinée, en tenant compte

\footnotetext{
${ }^{1}$ Robert Reix, Conférence invitée à l'Université Saint-Joseph, Beyrouth, Liban (28/10/2002)

${ }^{2}$ https://www.haussmann-es.com/etude-chief-digital-officers-2017/06-Les-CDO-du-CAC40
} 
des fonctionnalités matérielles des médias constituant le portefeuille des moyens de communication et de leur appropriation par les utilisateurs, ils revisitent ainsi la théorie du millefeuille.

Fadia Bahri Korbi présente un article sur la transformation numérique des relations interorganisationnelles en analysant la décision d'adopter des systèmes d'information inter-organisationnels (SIIO) au sein de partenariats stratégiques. Avec l'étude de dix cas d'alliances asymétriques entre des entreprises tunisiennes et européennes, elle révèle les facteurs qui influencent la décision d'adoption en fonction des caractéristiques de l'alliance asymétrique.

\section{BIBLIOGRAPHIE}

Constantinides, P., Henfridsson, O., \& Parker, G. G. (2018). Platforms and Infrastructures in the Digital Age. Information Systems Research, 29(2): 381-400.

Denner, MS., Püschel, L.C. \& Röglinger, M. (2018). How to Exploit the Digitalization Potential of Business Processes. Business and Information System Engineering, 60(4): 331-349.

Eden, R., Burton-Jones, A., Casey, V., \& Draheim, M. (2019). Digital Transformation Requires Workforce Transformation. MIS Quarterly Executive, 18(1): 1-17.
Faraj, S., Pachidi, S., \& Sayegh, K. (2018). Working and organizing in the age of the learning algorithm. Information and Organization, 28(1): 62-70.

Kappelman, L., Johnson, V., Torres, R., Maurer, C., \& McLean, E. (2019). A study of information systems issues, practices, and leadership in Europe. European Journal of Information Systems, 28(1): 26-42.

Kauffman, R. J., \& Weber, T. A. (2018). Special Section: The Digital Transformation of Vertical Organizational Relationships. Journal of Management Information Systems, 35(3): 837-839.

Li, L., Su, F., Zhang, W., \& Mao, J. Y. (2018). Digital transformation by SME entrepreneurs: A capability perspective. Information Systems Journal, 28(6): 1129-1157.

Markus, M. L. (2019). Digital Transformation and How it Happens, $24^{e}$ conférence de l'AIM.

Nambisan, S., Lyytinen, K., Majchrzak, A., \& Song, M. (2017). Digital Innovation Management: Reinventing Innovation Management Research in a Digital World. MIS Quarterly, 41(1): 223-238.

Orlikowski, W. J., \& Scott, S. (2016). Digital work: A research agenda, in A Research Agenda for Management and Organization Studies, B. Czarniawska (ed.), Cheltenham, UK: Edward Elgar: 88-95.

Pigneur, Y. (2019). Innover et repenser son modèle d'affaires, $24^{e}$ conférence de l'AIM. 